\title{
Trends, predictors and outcomes of ischemic stroke and intracranial hemorrhage in patients with a left ventricular assist device
}

\author{
Muhammad Shahreyar ${ }^{1,2 *}$, Tamunoinemi Bob-Manuel ${ }^{1 *}$, Rami N. Khouzam ${ }^{1,2}$, Mohammad W. Bashir ${ }^{3}$, \\ Samian Sulaiman ${ }^{4}$, Oluwaseun Akinseye ${ }^{1,2}$, Arindam Sharma ${ }^{1}$, April Carter ${ }^{1}$, Samuel Latham ${ }^{1}$, Sanjay \\ Bhandari $^{4}$, Arshad Jahangir ${ }^{5,6}$ \\ ${ }^{1}$ Department of Medicine, University of Tennessee Health Science Center, Memphis, TN, USA; ${ }^{2}$ Division of Cardiology, University of Tennessee \\ Health Science Center, Memphis, TN, USA; ${ }^{3}$ University of Gdansk, Gdansk, Poland; ${ }^{4}$ Department of Internal Medicine, Medical College of \\ Wisconsin, Milwaukee, WI, USA; ${ }^{5}$ Aurora Cardiovascular Services, Aurora St. Luke's Medical Center, Milwaukee, WI, USA; ${ }^{6}$ Center for Integrative \\ Research on Cardiovascular Aging (CIRCA), Aurora St. Luke's Medical Center, Aurora Health Care, Milwaukee, WI, USA \\ Contributions: (I) Conception and design: A Jahangir; (II) Administrative support: None; (III) Provision of study materials or patients: M Shahreyar; \\ (IV) Collection and assembly of data: A Jahangir, M Shahreyar; (V) Data analysis and interpretation: A Jahangir, M Shahreyar, T Bob-Manuel; (VI) \\ Manuscript writing: All authors; (VII) Final approval of manuscript: All authors. \\ *These authors contributed equally to this work. \\ Correspondence to: Arshad Jahangir, MD. Aurora Cardiovascular Services, Aurora St Luke's Medical Center, Aurora Health Care, Milwaukee, WI \\ 53215, USA. Email: publishing44@aurora.org.
}

Background: Patients with a left ventricular assist device (LVAD) are at a higher risk of ischemic stroke (IS) and intracranial hemorrhage (ICH). There is limited data available on risk factors and outcomes associated with IS and ICH in LVAD patients.

Methods: All patients $>18$ years of age with an LVAD were identified based on the U.S. Nationwide Inpatient Sample (NIS) database from the year 2007 to 2011. Patients with a discharge diagnosis of IS were compared to those without IS. In a separate analysis, patients with a discharge diagnosis of ICH were compared to patients without ICH. Trends, predictors and outcomes of IS and ICH were analyzed using a multivariate regression model.

Results: Out of 17,323 discharges with a primary diagnosis of heart failure with LVAD, 624 (3.6\%) patients had a co-diagnosis of IS and 387 (2.2\%) had a co-diagnosis of ICH. From 2007 to 2011, the discharge diagnosis of heart failure with LVAD increased from 946 to 5,540, but the proportion of patients with IS remained about 3.4\%, while the incidence of $\mathrm{ICH}$ decreased from $3.8 \%$ in 2007 to a plateau of around $2.2 \%$ in the following years. After adjusting for potential confounders, increasing Charlson Comorbidity Index (CCI) score was an independent predictor of IS and ICH. In-hospital mortality was four-fold higher in the IS group (odds ratio: 4.2; 95\% CI: 2.3-7.6; $\mathrm{P}<0.0001$ ) and 18-fold higher in the ICH group (OR: 18; 95\% CI: 9-34, $\mathrm{P}<0.0001)$. Renal disease (OR: 5.3; CI: 1.3-22.1; P=0.02), liver disease (OR: 4.9; CI: 1.1-21.2; $\mathrm{P}=0.03$ ) and abnormal coagulation profile (OR: 4.8; CI: 1.6-14.4; $\mathrm{P}=0.01$ ) were independent predictors of mortality in LVAD patients with IS. Presence of diabetes mellitus (OR 4.3, $\mathrm{P}=0.1$ ) and liver disease (or 2.8, $\mathrm{P}=0.2$ ) showed trends towards predicting mortality in LVAD patients with ICH but did not reach statistical significance.

Conclusions: Increasing comorbidity burden significantly increases the risk of both IS and ICH with LVAD. In our cohort, the incidence of IS and ICH increases the mortality 4- and 18-fold, respectively. Renal disease, liver disease and abnormal coagulation profile were independent predictors of mortality in LVAD patients with IS. 
Keywords: Left ventricular assist device (LVAD); ischemic stroke (IS); intracranial hemorrhage (ICH); mortality; heart failure

Submitted Nov 20, 2017. Accepted for publication Dec 02, 2017.

doi: $10.21037 /$ atm.2017.12.23

View this article at: http://dx.doi.org/10.21037/atm.2017.12.23

\section{Introduction}

In the United States (US), the estimated total cost of heart failure was $\$ 30.7$ billion in 2012 (1). Approximately 6.5 million Americans $\geq 20$ years of age suffer from heart failure (1). Advanced systolic heart failure carries an exceedingly poor life expectancy of merely 6 months (2). With improvement in technology and use of implantable mechanical circulatory support devices, the clinical course of advanced heart failure patients in terms of both quality and quantity of life has changed considerably. Over 1,500 left ventricular assist devices (LVADs) are placed in the US annually, and due to the increasing prevalence of advanced heart failure and transplant donor heart shortage this number is expected to rise (3). In addition to being a bridge to transplantation, LVADs are being utilized as destination therapy in those with advanced age and or multiple comorbidities who are ineligible for cardiac transplantation (4-6). Despite advancements in patient care, the longterm use of LVAD poses new challenges including a high incidence of cerebrovascular accidents (CVAs), infections and bleeding that contribute to morbidity and mortality in these patients which could potentially be reduced with better understanding of risk factors and improvement in patient care (5).

CVAs, in particular hemorrhagic CVAs, remain one of the leading causes of morbidity and mortality associated with LVADs. It is also the primary reason for withdrawal from cardiac transplantation waiting list in LVAD-supported patients. The incidence of hemorrhagic and ischemic strokes (ISs) in the first several months following LVAD placement ranges between $8 \%$ to $25 \%$ (7). The altered thrombotic profile caused by contact of blood with biomaterials used in CF-LVAD and the concurrent need for postoperative anticoagulation with both antiplatelet and anticoagulation (8-10) predispose to ischemic and hemorrhagic strokes. The risk factors, characteristics and outcomes of stroke in patients with LVAD have not been well established. The goal of this study is to examine the trends, predictors, and outcomes of ischemic and hemorrhagic stroke in patient with LVAD.

\section{Methods}

\section{Search strategy}

\section{Patient selection}

Information was obtained from the U.S. Nationwide Inpatient Sample (NIS) database from 2007 to 2011. All patients with LVAD were included. The International Classification of Diseases, Ninth Revision, Clinical Modification (ICD-9-CM) codes were used to identify particular diseases, complications, and inpatient procedures. Patients with a co-diagnosis of IS were compared to patients without any diagnosis of stroke, and patients who had a codiagnosis of ICH were compared to patients without any diagnosis of stroke. We applied the Charlson Comorbidity Index (CCI) as a predictive tool for incidence of either stroke diagnosis. Trends, predictors and outcomes of IS and ICH were analyzed using a multivariate regression model.

The NIS is sponsored by Agency for Healthcare Research and Quality (AHRQ) of the US Department of Health and Human Services. The NIS represents approximately $20 \%$ sample of all discharges in nonfederal US hospitals ${ }^{1}$.

\section{Exclusion criteria}

Patients younger than 18 years of age were excluded. Those who were discharged to another hospital were excluded to prevent single patients from being represented multiple times. Observations with any missing variables (except for the ethnicity) were excluded.

\section{Statistical analysis}

We compared baseline characteristics of patients with a co-

\footnotetext{
${ }^{1}$ https://www.hcup-us.ahrq.gov/nisoverview.jsp
} 


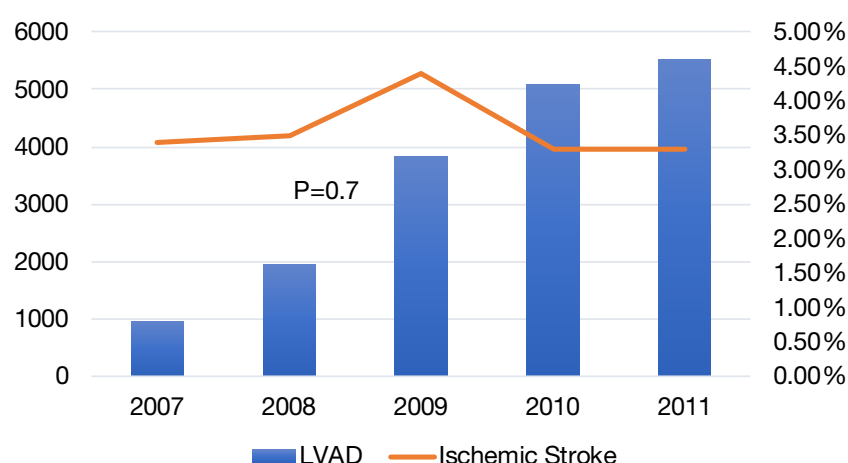

Figure 1 Yearly trend in admission with patients with LVAD and IS in LVAD. LVAD, left ventricular assist device; IS, ischemic stroke.

diagnosis of IS to patients without any diagnosis of stroke and patients with a diagnosis of ICH to patients without any diagnosis of stroke. Categorical variables were summarized with the use of frequencies and percentages. Chi-squared test was used to compare categorical variables. A two-sided $\mathrm{P}$ value $<0.05$ was used to assess statistical significance. Multivariate regression analysis was done with IS as a binary dependent variable and baseline patient characteristicsincluding demographics (age, gender, race), having LVAD in previous hospitalization, being on heart transplant waiting list, Charlson Comorbidities index, individual comorbidities (including hypertension, myocardial infarction, peripheral vascular disease, chronic lung disease, diabetes mellitus, liver disease, renal disease, cancer and valvular disease), abnormal coagulation profile and the year of LVAD implantation as independent covariates. Similar multivariate regression analysis was done for $\mathrm{ICH}$ as a binary dependent variable along with above-mentioned independent variables. A list of ICD-9-CM and Clinical Classifications Software codes used to identify comorbidities is provided online in Supplementary.

Univariate (Chi Square) and multivariate regression analysis was done to investigate the impact of IS on mortality in patients with LVAD. Variables included in addition to IS, age, gender, race, LVAD implantation in previous hospitalization, being on heart transplant list, CCI, abnormal coagulation profile, the year of LVAD implantation and individual comorbidities (including hypertension, myocardial infarction, peripheral vascular disease, chronic lung disease, diabetes mellitus, liver disease, renal disease, cancer and valvular disease). Similar multivariate regression analysis was done to investigate the impact of ICH on mortality in patients with LVAD. Data were analyzed using SAS statistical software package Version 9.4 (SAS Institute Inc., Cary, NC, USA).

\section{Results}

\section{IS}

\section{Characteristics of patients with LVAD hospitalized with or without IS}

Out of a total of 17,323 patients with advanced heart failure and LVAD who were hospitalized during the study period, 624 (3.6\%) patients were hospitalized with IS after LVAD placement. Although the total number of patients with LVAD and percentage of all hospitalization gradually increased from $0.5 \%$ in 2007 to $4.5 \%$ in 2011 , the overall percentage of LVAD patients with IS has been similar over these years, around $3.5 \%$ of all patients with LVAD (Figure 1). The baseline characteristics of the 624 patients who had IS and 16,699 patients with LVAD without IS are summarized in Table 1 . There were no significant differences between these two groups in terms of age, sex, racial or ethnic distribution, those awaiting cardiac transplant, major comorbidities, those with abnormal coagulation profile or the year of LVAD placement. The major difference between the LVAD patients hospitalized with or without IS was in the number of advanced critical comorbidities present as calculated by the CCI, with a much greater proportion of patients with a CCI greater than or equal to 4 in IS compared to non-stroke patients ( $48 \%$ vs. $18 \%, \mathrm{P}<0.001$; Table 1). Patients with IS had a lower prevalence of chronic lung disease compared to those hospitalized without IS (25\% vs. $36 \%, \mathrm{P}=0.01$ ).

\section{Risk factors for IS in hospitalized patients with LVAD}

Factors associated with increased risk for IS in patients hospitalized with LVAD were determined with multivariate regression analysis of baseline characteristics, including individual comorbidities, CCI reflecting multiple comorbidities, presence of abnormal coagulation profile, and the year of LVAD placement (2007 vs. 2011). None of the individual comorbidities, including those associated with the risk of stroke for patients with $\mathrm{AF}$, such as advanced age, female sex, history of hypertension, diabetes or abnormal coagulation profile or other comorbidities (Table 2) were associated with risk of IS. The risk of IS was however lower in White compared to Hispanics (OR: 0.239; 95\% CI: $0.073-0.782 ; \mathrm{P}=0.02)$, with a statistically non-significant 
Table 1 Baseline characteristics of patients with LVAD with or without IS after LVAD implantation

\begin{tabular}{|c|c|c|c|}
\hline Demographics & LVAD without IS $(\mathrm{N}=16,699)$ & LVAD with IS (N=624) & $P$ values \\
\hline Age category (years) & & & 0.6 \\
\hline$\geq 65$ & $4,763[28]$ & $165[26]$ & \\
\hline Female $^{a}$ & $4,164[25]$ & $164[26]$ & 0.7 \\
\hline White & $9,094[54]$ & 366 [59] & \\
\hline Black & $3,379[20]$ & $108[17]$ & \\
\hline Hispanic & $876[5]$ & $10[2]$ & \\
\hline Waiting for heart transplant & $1,763[11]$ & $55[9]$ & 0.6 \\
\hline \multicolumn{4}{|l|}{ Charlson Comorbidity Index } \\
\hline $0-1$ & $3,721[22]$ & 9 [1] & \\
\hline 2 & $5,464[33]$ & $111[18]$ & \\
\hline 3 & $4,538[27]$ & 207 [33] & \\
\hline $4+$ & $2,976[18]$ & $297[48]$ & $<0.0001$ \\
\hline Abnormal coagulation profile & $2,228[13]$ & $107[17]$ & 0.3 \\
\hline Chronic lung disease & $6,069[36]$ & $153[25]$ & 0.01 \\
\hline DM & $5,344[32]$ & 184 [29] & 0.6 \\
\hline Liver disease & $1,316[8]$ & 68 [11] & 0.2 \\
\hline Renal disease & $5,709[34]$ & $167[27]$ & 0.1 \\
\hline Year & & & 0.7 \\
\hline 2007 & 914 [6] & $32[5]$ & \\
\hline 2008 & $1,871[11]$ & $68[11]$ & \\
\hline 2009 & $3,646[22]$ & $169[27]$ & \\
\hline 2010 & $4,911[29]$ & $170[27]$ & \\
\hline 2011 & $5,356[32]$ & $184[29]$ & \\
\hline
\end{tabular}

a, 4 observations with missing values for sex; ${ }^{b}$, can range from 0 to 17 . LVAD, left ventricular assist device; IS, ischemic stroke; HTN, hypertension; AF, atrial fibrillation; PVD, peripheral vascular disease; DM, diabetes mellitus. 
Table 2 Predictors of ischemic stroke with odds ratio and 95\% confidence interval for IS in patients with LVAD on multivariate analysis

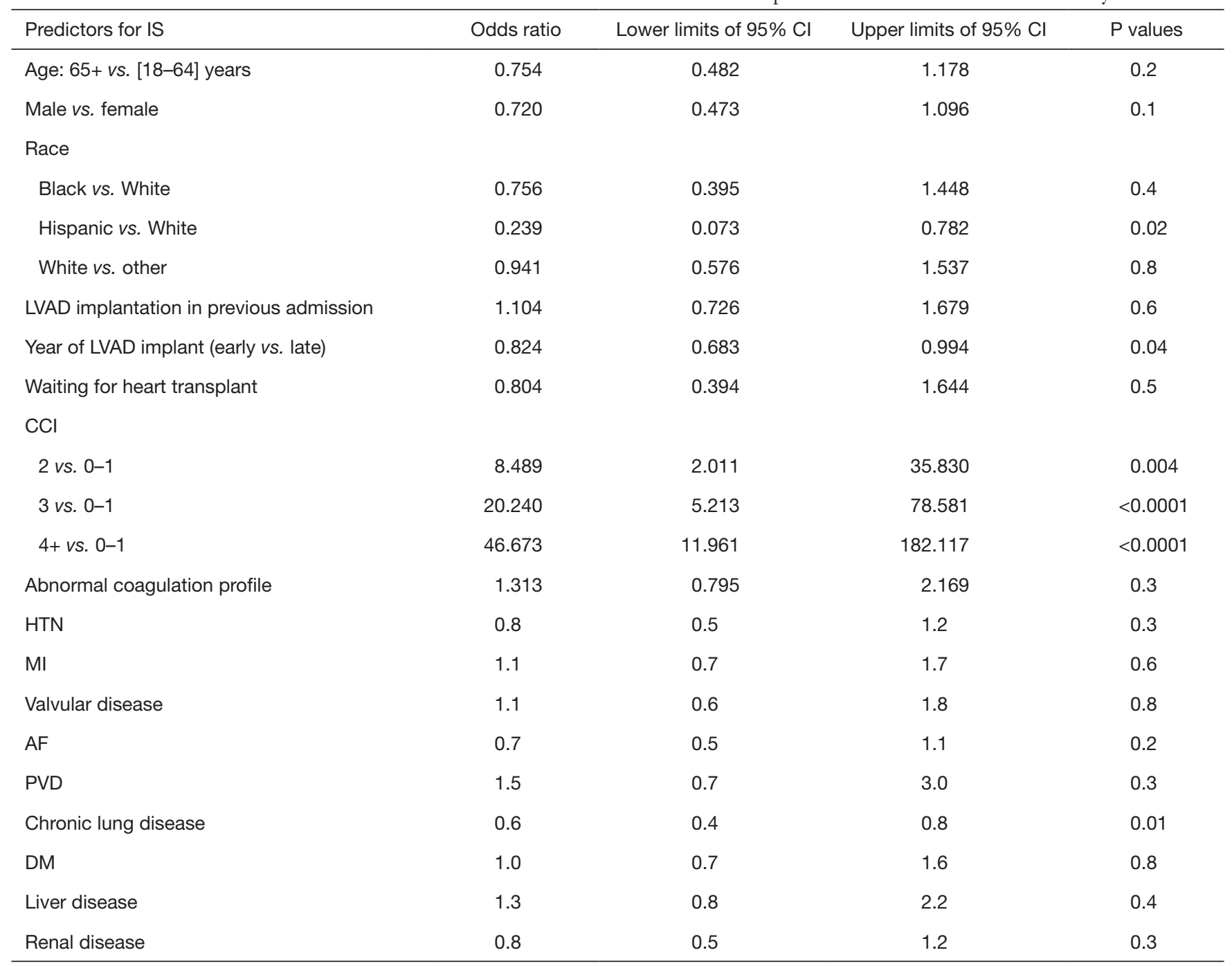

LVAD, left ventricular assist device; IS, ischemic stroke; CCI, Charlson Comorbidity Index; HTN, hypertension; AF, atrial fibrillation; PVD, peripheral vascular disease; DM, diabetes mellitus.

trend toward a higher risk of IS in white compared to black (Table 2). There was a strong correlation with the number of critical illnesses as reflected by CCI with the risk of IS increasing 8.5 folds in those with CCI 2 compared to $0-1$, to 20 and 47 folds in those with CCI 3 and 4 and greater, respectively (Table 2, Figure 2).

\section{Mortality and predictors of in-hospital death in LVAD patients hospitalized with IS}

The in-hospital mortality of patients with IS was 4.8 folds higher $(\mathrm{OR}=4.8 ; 95 \% \mathrm{CI}: 2.8-8.2 ; \mathrm{P}<0.001)$ than those without IS. On multivariate analysis, factors associated with higher mortality in LVAD patients with IS included advanced liver disease, renal disease, cancer and abnormal coagulation profile (Table 3).

\section{Intracranial bemorrbage (ICH)}

\section{Characteristics of patients with LVAD with or without ICH}

Out of the total of 17,323 patients with LVAD who were hospitalized during the study period, 387 patients were hospitalized with $\mathrm{ICH}$. There was a significant drop in incidence of ICH from $3.9 \%$ in 2007 to $2.2 \%$ in 2008 with a plateau thereafter (Figure 3). ICH was subdivided into five different categories with intracerebral hemorrhage 


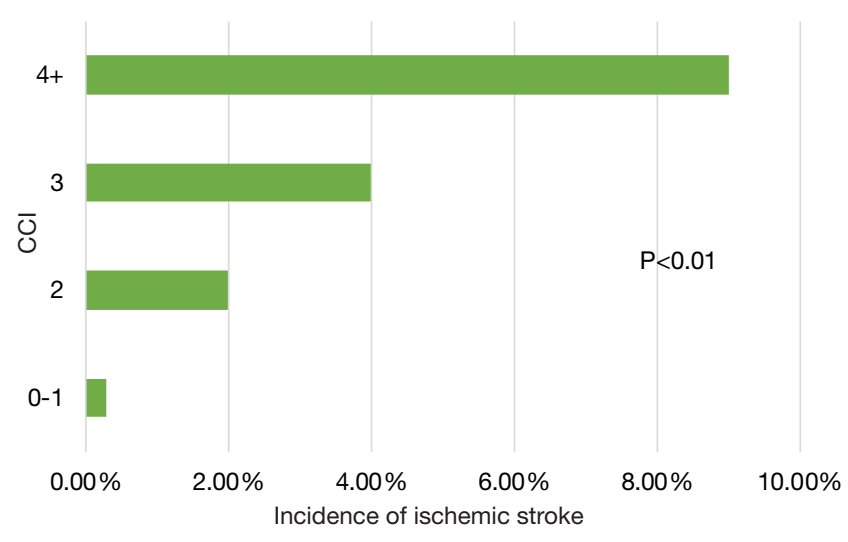

Figure 2 Incidence of ischemic stroke in patients hospitalized with left ventricular assist device implanted for advanced heart failure by Charlson Comorbidity Index (CCI). being the most common (47.4\%) followed by subarachnoid hemorrhage (26\%), subdural hemorrhage $(12.1 \%)$ and extradural hemorrhage (3.2\%) (Table 4). In $11 \%$ of patients ICH was unspecified.

The baseline characteristics of the 387 patients who had ICH and 16,935 patients with LVAD without ICH are summarized in Table 5. There were no significant differences between these two groups in terms of age, sex, racial or ethnic distribution, those awaiting cardiac transplant or year of LVAD implant. The LVAD patients who were hospitalized with ICH were more likely to have a higher number of advanced critical illnesses as calculated by the CCI of 3 (35\% vs. 27\% non-ICH patients) or 4 and higher (36\% vs. 19\%; $\mathrm{P}<0.001$, Table 5). Patients with ICH were more likely to have liver disease (16\% vs. $8 \%$,

Table 3 Independent predictors of mortality in LVAD patients with ischemic stroke (multivariate analysis) ${ }^{\mathrm{a}}$

\begin{tabular}{|c|c|c|c|c|}
\hline Predictors & Odds ratio & Lower limits of $95 \% \mathrm{Cl}$ & Upper limits of $95 \% \mathrm{Cl}$ & $P$ values \\
\hline Age: $65+$ vs. [18-64] years & 1.655 & 0.652 & 4.200 & 0.3 \\
\hline \multicolumn{5}{|l|}{ Race } \\
\hline Female vs. male & 0.776 & 0.324 & 1.857 & 0.6 \\
\hline White vs. Black & 0.664 & 0.145 & 3.051 & 0.6 \\
\hline LVAD implantation in previous admission & 1.295 & 0.516 & 3.252 & 0.6 \\
\hline Year of LVAD implant (2007 vs. 2011) & 0.700 & 0.433 & 1.131 & 0.1 \\
\hline Waiting for heart transplant & 0.736 & 0.188 & 2.885 & 0.7 \\
\hline CCl: $3+$ vs. $0-2$ & 3.245 & 0.550 & 19.138 & 0.2 \\
\hline Valvular disease & 1.362 & 0.280 & 6.620 & 0.7 \\
\hline AF & 0.8 & 1.7 & 0.5 & 0.1 \\
\hline PVD & 0.441 & 0.056 & 3.440 & 0.4 \\
\hline Chronic lung disease & 0.745 & 0.148 & 3.742 & 0.7 \\
\hline DM & 0.744 & 0.200 & 2.770 & 0.7 \\
\hline Liver disease & 4.908 & 1.132 & 21.277 & 0.03 \\
\hline Renal disease & 5.368 & 1.302 & 22.134 & 0.02 \\
\hline
\end{tabular}

${ }^{a}$, all Hispanic patients with IS ( $\mathrm{N}=10$ ) died. None of the patients with IS and CCl of 0-1 (lower CCI) died. LVAD, left ventricular assist device; $\mathrm{CCl}$, Charlson Comorbidity Index; HTN, hypertension; AF, atrial fibrillation; PVD, peripheral vascular disease; DM, diabetes mellitus. 


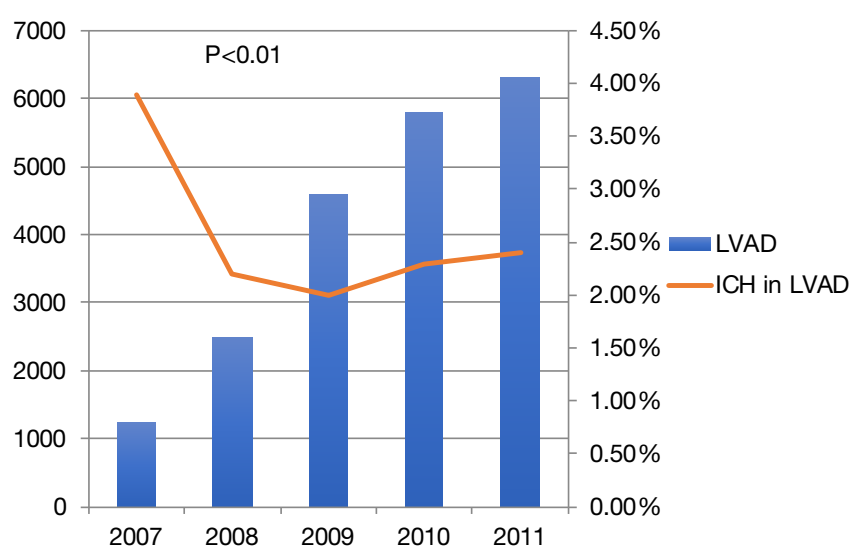

Figure 3 Yearly trend in admission with patients with LVAD and ICH in LVAD. LVAD, left ventricular assist device; ICH, intracranial hemorrhage.

Table 4 Frequency distribution of intracranial hemorrhage subtypes

\begin{tabular}{lc}
\hline Intracranial hemorrhage subtype & Frequencies (\%) \\
\hline Subarachnoid hemorrhage & $114(26.0)$ \\
Intracerebral hemorrhage & $208(47.4)$ \\
Extradural hemorrhage & $14(3.2)$ \\
Subdural hemorrhage & $53(12.1)$ \\
Unspecified intracranial hemorrhage & $50(11.4)$ \\
Total & $439^{\mathrm{a}}$ \\
\hline
\end{tabular}

a, 52 were overlapping diagnosis.

$\mathrm{P}=0.05)$. Surprisingly, the prevalence of hypertension, diabetes and valvular heart disease was significantly lower in LVAD patients hospitalized with ICH than those without ICH (Table 5). There was no significant difference in the proportion of patients with abnormal coagulation profile between the two groups.

\section{Risk factors for ICH in hospitalized patients with LVAD} Factors associated with increased risk for ICH in LVAD patients were identified by multivariate regression analysis of baseline characteristics and are summarized with odds ratio and 95\% confidence interval in Table 6. Surprisingly, the youngest age group (18-35 years) was at the highest risk for ICH when compared to the youngadult (36-50 years: OR: 0.284 , 95\% CI: $0.117-0.690$, $\mathrm{P}=0.01$ ), middle-age group (51-65 years) and the elderly age group ( $>65$ years). In contrast to IS, rising CCI increased the risk of ICH by 8 folds only if the CCI score was $>3$. None of the individual comorbidities, were associated with risk of ICH but surprisingly DM, PVD and COPD were associated with a decreased risk of ICH. In addition, patients who received LVAD in year 2007 where at higher risk of ICH compared to those who were implanted in 2011.

\section{Mortality and predictors of in-hospital death in LVAD patients hospitalized with ICH}

The in-hospital mortality of patients with ICH was 18 folds higher $(\mathrm{OR}=18$; 95\% CI: 9-34; $\mathrm{P}<0.001)$ than those without ICH. On multivariate analysis, no significant associations between individual comorbidities and mortality could be identified (Table 7).

\section{Discussion}

The use of LVAD has become a standard part of therapy for patients with end-stage heart failure, employed either as a bridge to transplantation or destination therapy in patients who are not candidates for transplantation. Despite tremendous impact on improving quality of life, morbidity and mortality of advanced heart failure patients, LVAD therapy is associated with challenges, such as increased risk of thromboembolic complications and life-threatening bleeding, partly related to the use of anticoagulants and underlying comorbidities are major limitations. Stroke, both ischemic and hemorrhagic, remains a serious challenge associated with significantly increased morbidity and mortality (11-13). To our knowledge, the present study is the largest to use discharge diagnoses from the National Inpatient Sample (NIS) database to validate various trends, predictors and outcomes of strokes in LVAD patients (4-6). While most studies in literature have analyzed data from single/multi-center registries and cohorts, our study measures risk factors and outcomes through discharge diagnoses of National Inpatient Survey database, thus finding and validating findings through a diametrically opposite experiment design. Another strength of our study is that it employs data sourced from multiple centers nationwide, and thus has a fair degree of generalizability.

Over the 5 years period analyzed, the overall hospitalization of patients with LVAD has increased as more patients with advanced heart failure are receiving LVAD. In this database, the number of hospitalization for patients with LVAD in the year 2007 was less than a thousand, which has increased by more than fivefold in the subsequent 5 years to more than 5,500 hospitalizations in 2011 . 
Table 5 Baseline characteristics of patients with left ventricular assist device with or without ICH after LVAD implantation (without ICH vs. LVAD with ICH)

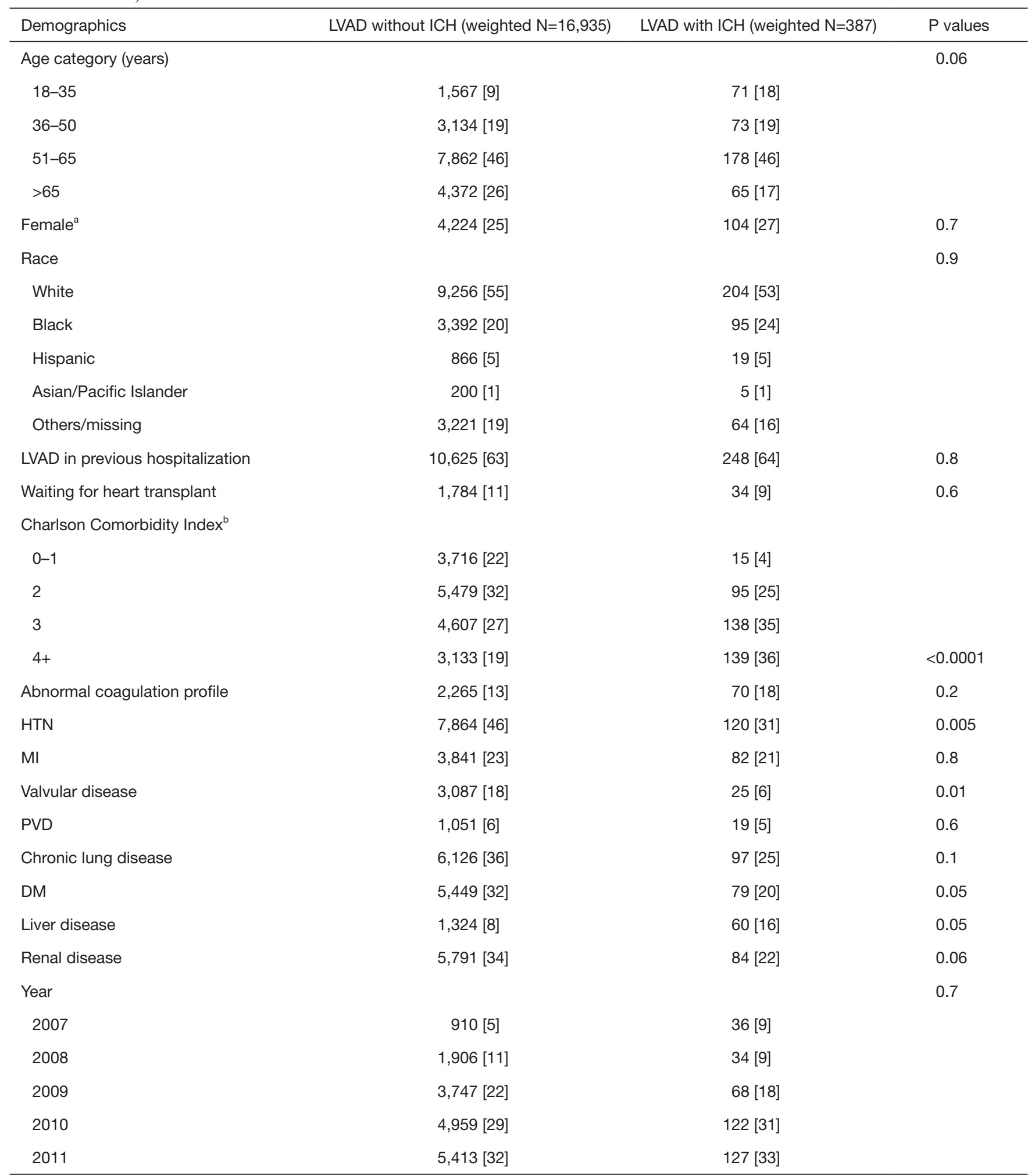

a , 4 observations with missing values for sex; ${ }^{b}$, can range from 0 to $17 . \mathrm{ICH}$, intracranial hemorrhage; LVAD, left ventricular assist device; HTN, hypertension; AF, atrial fibrillation; PVD, peripheral vascular disease; DM, diabetes mellitus. 
Table 6 Predictors of intracranial hemorrhage with odds ratio and 95\% confidence interval for ICH in patients with LVAD (multivariate analysis)

\begin{tabular}{|c|c|c|c|c|}
\hline Predictors of $\mathrm{ICH}$ & Odds ratio & Lower limits of $95 \% \mathrm{Cl}$ & Upper limits of $95 \% \mathrm{Cl}$ & $P$ values \\
\hline [18-35] vs. [51-65] & 2.4 & 1.2 & 4.7 & 0.01 \\
\hline [36-50] vs. [51-65] & 0.9 & 0.4 & 1.7 & 0.6 \\
\hline $66+$ vs. [51-65] & 0.6 & 0.3 & 1.2 & 0.1 \\
\hline \multicolumn{5}{|l|}{ Race } \\
\hline Black vs. White & 1.406 & 0.676 & 2.925 & 0.4 \\
\hline Hispanic vs. White & 0.957 & 0.268 & 3.416 & 0.9 \\
\hline White vs. other & 1.178 & 0.625 & 2.221 & 0.6 \\
\hline 2 vs. 3 & 0.075 & 0.043 & 0.130 & $<0.0001$ \\
\hline 4 vs. 3 & 8.303 & 3.959 & 17.412 & $<0.0001$ \\
\hline Abnormal coagulation profile & 1.415 & 0.653 & 3.064 & 0.4 \\
\hline HTN & 0.826 & 0.463 & 1.473 & 0.5 \\
\hline PVD & 0.118 & 0.038 & 0.363 & 0.0002 \\
\hline Chronic lung disease & 0.073 & 0.036 & 0.149 & $<0.0001$ \\
\hline DM & 0.053 & 0.024 & 0.116 & $<0.0001$ \\
\hline Liver disease & 0.936 & 0.634 & 1.347 & 0.8 \\
\hline 2007 vs. 2011 & 2.831 & 1.053 & 7.616 & 0.04 \\
\hline 2008 vs. 2011 & 1.211 & 0.578 & 2.538 & 0.6 \\
\hline 2009 vs. 2011 & 0.948 & 0.410 & 2.194 & 0.9 \\
\hline 2010 vs. 2011 & 1.196 & 0.648 & 2.206 & 0.6 \\
\hline
\end{tabular}

LVAD, left ventricular assist device; ICH, intracranial hemorrhage; $\mathrm{CCl}$, Charlson Comorbidity Index; HTN, hypertension; PVD, peripheral vascular disease; DM, diabetes mellitus.

Despite the increase in the number of LVAD implants, the proportion of patients admitted with IS has not changed significantly, with approximate $3.4 \%$ per year incidence. Across various studies, the incidence of IS has been found to range between $6 \%$ to $10.6 \%(11,14)$, and the incidence of ICH has been reported in the range between 3\% to $8.4 \%$ $(11,15)$. In our study, the prevalence of IS was $3.4 \%$, and
ICH was $2.2 \%$. The lower incidence rates in our study are likely due to the limited timeline of stroke captured only during the index hospitalization.

The likelihood of IS in the hospitalized patients increased with the increase in the number of co-existing comorbidities present as reflected by a greater proportion of patients with $\mathrm{CCI} \geq 4$. CCI is the most commonly used comorbidity index 
Table 7 Predictors of mortality in ICH in LVAD on multivariate analysis

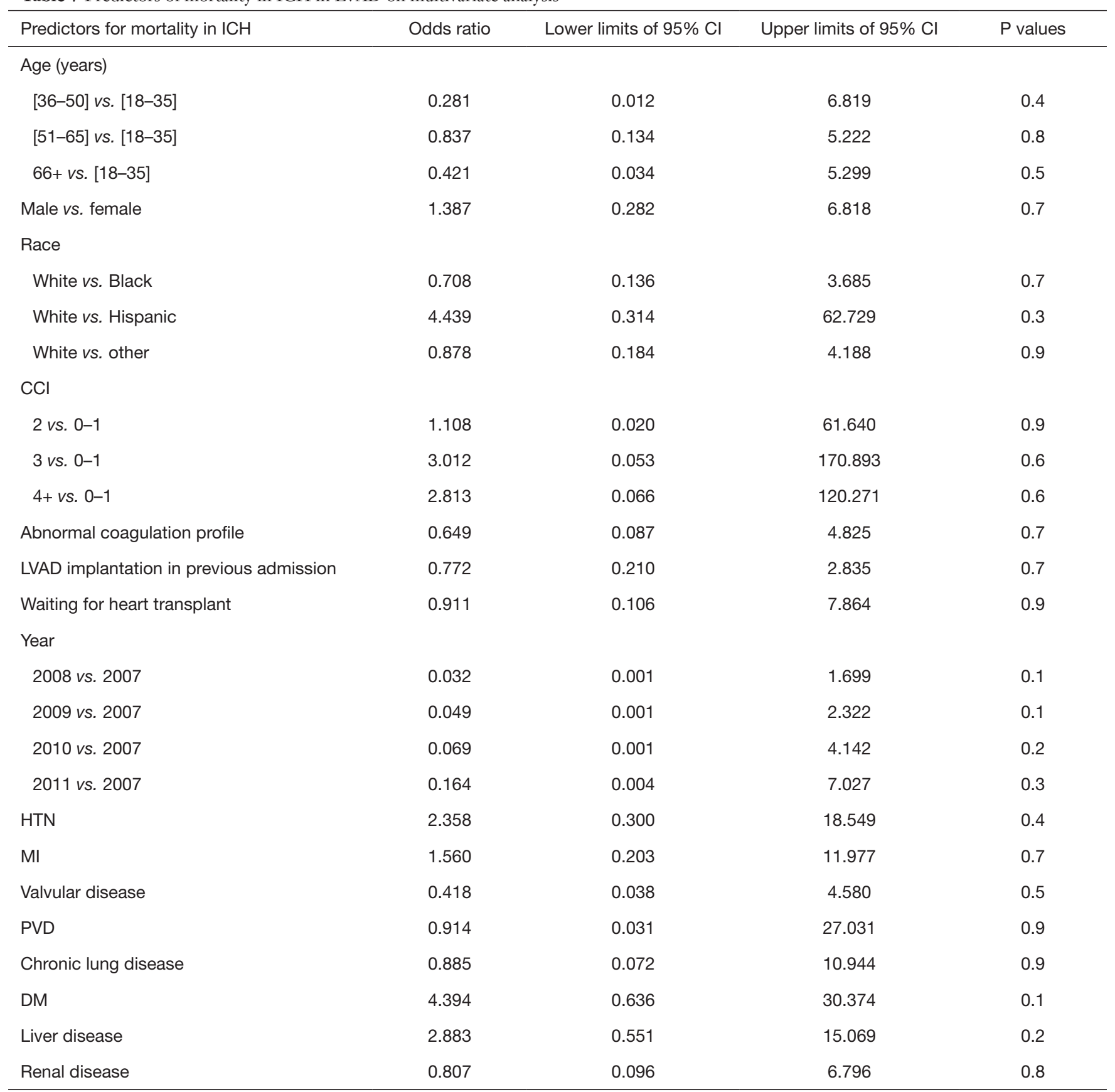

$\mathrm{ICH}$, intracranial hemorrhage; LVAD, left ventricular assist device; CCI, Charlson Comorbidity Index; HTN, hypertension; MI, myocardial infarction; PVD, peripheral vascular disease; DM, diabetes mellitus.

$(16,17)$ composed of 19 comorbid conditions including diabetes with diabetic complications, congestive heart failure, myocardial infarction, peripheral vascular disease, cerebrovascular disease, chronic pulmonary disease, connective tissue disease, mild, moderate or severe liver disease, ulcer disease, hemiplegia, moderate or severe renal disease, leukemia, lymphoma, non-metastatic and metastatic tumor, and acquired immunodeficiency syndrome (AIDS), each comorbid condition weighted separately on the basis of its association with 1-year mortality. Higher scores 
indicate greater comorbidities and a higher risk of dying at 1 year (17), with CCI been verified as a valid method of accurately predicting outcomes and risk of death in many conditions $(18,19)$ as was found in our cohort of LVAD patients with IS or ICH. A CCI of $\geq 2$ was an independent predictor of both IS and ICH in LVAD patients. Increasing CCI scores were also an independent predictor of mortality in patients with ICH, but not in those with IS.

Although, combined comorbidities predicted the risk of IS and ICH, individual comorbidities, including those known to increase the risk of stroke and thromboembolism in patients with or without $\mathrm{AF}$, such as advanced age, female sex, presence of hypertension, diabetes and valvular heart disease were not independently associated with IS or ICH in these patients with heart failure who were already on chronic anticoagulation. Although, data regarding abnormal coagulation profile is available in the NIS database, the information about anticoagulation time in therapeutic range at the time of IS is not available and it is difficult to ascertain from this administrative database if the stroke occurred due to subtherapeutic INR or other reasons. We tested several patient factors with whom an association was seen in previous studies, such as diabetes $(11,12,20)$, hypertension $(13,20)$, female gender $(11,21)$ and tobacco use (20). Our study failed to find any significant association with these factors. Many of these factors are traditional risk factors for stroke in general, and these may not play as important a role in early strokes in LVAD patients.

Another important finding of this study, which is consistent with other reports $(11,22)$, is the higher incidence of ICH in younger patients with LVAD. Those younger than 35 years of age were at greater risk of developing ICH than older age groups. It is unclear why younger patients are more prone to $\mathrm{ICH}$ as it seems almost counterintuitive with more comorbidities expected in older patients. We hypothesize that younger patients with end stage heart failure undergoing LVAD placement are outliers: likely having severe disease in more than one organ system, for example genetic and metabolic congenital abnormalities all increasing their risk for ICH compared to older patients likely having only a primary cardiac failure. Alternatively, younger patients may also exhibit non-compliance with fluctuating anticoagulation that may increase the risk of bleeding or thromboembolism. A study looking specifically at this subgroup is necessary to identify the causes of higher stroke rates in this subgroup.

Interestingly, LVAD patients of Hispanic origin were significantly less likely to develop an IS as compared to
Whites. In general, the stroke rates in Hispanics have been found to be significantly higher than whites in several studies $(23,24)$. In the LVAD population, races other than Whites and African Americans have not been analyzed in most studies, likely due to the smaller number of such patients. A large study in 2016 using Administrative Claims data did not find any association between race and LVAD stroke rates. Our finding suggests that further sub-analysis in different racial categories is warranted that may help elucidate further trends and predictors of stroke in these subgroups.

Both IS and ICH are significant predictors of mortality in LVAD patients, accounting for an almost 4-fold increase in mortality in IS patients and an 18-fold increase in mortality in ICH patients. Given the devastatingly high impact strokes have on the mortality and quality of life of such patients, it is imperative to mitigate stroke risk to make LVADs more acceptable and safer to use in patients with advanced heart failure. The pathophysiology of increased stroke in LVAD implantation is related to the intrinsic propensity towards thrombogenicity as the LVAD foreign object encounters blood in circulation (8) and increased risk of bleeding which is expected with the use of chronic anticoagulation. Although, medications use that increases bleeding propensity is the most obvious etiology for bleeding in LVAD patients, several other mechanisms play a role in bleeding including, including the acquired Von Willebrand syndrome due to the effect of shear forces on clotting factors and platelet activation and aggregation (21).

While all patients with LVAD are typically on anticoagulation, data on coagulation status affecting stroke rates is conflicting at present. Studies have reported both sub-therapeutic $(13,20)$ and supra-therapeutic INR (15) levels as risk factors, and many studies including ours have failed to find any relationship between coagulation status and IS and ICH rates $(25,26)$. The pathogenesis of ICH and IS in LVAD patients is multifactorial, and coagulation status is likely to be just one factor among a multitude of reasons why LVAD patients experience stroke. Improvements in anticoagulation with even stricter INR control may reduce coagulation disorders. A multidisciplinary approach as suggested by Morgan et al. (13) that includes more frequent blood draws, as well as additional patient education focused on the impact of dietary intake and drug interaction on the potency of warfarin might be worth employing.

Finally, we saw a significant drop in incidence of ICH after 2007. In is important to note that this coincides with the timing of shift in the use of LVAD with pulsatile-flow to continuous-flow support (27). There is a paucity of studies 
comparing the risk of ICH in pulsatile versus continuous flow LVAD. Even though in a prospective trial comparing clinical outcomes and adverse events in continuous versus pulsatile-flow LVAD, Slaughter et al. did not find a statistically significant difference in ICH it is important to note that the study was not adequately powered to assess incidence of ICH (28).

Being a retrospective analysis, the study has several limitations. First, our use of administrative claims data means that clinical variables such as stroke severity, antiplatelet and anticoagulant medication use, laboratory values that may reflect pro-coagulant or anticoagulant status, imaging findings, and other dynamic measures of outcomes were not available hence could not be included in the analysis. Second, bias inherent in retrospective analysis (29) of large population-based studies by searching hospital-originated databases and registries could not be avoided. Hence, the investigators are highly dependent on the skill of initial data entry by non-medical professionals, and the completeness of database queries. Individual records could not be verified nor double-checked and only preexisting variables could be analyzed. The impact of these limitations must be balanced against the large sample size and the associated benefits in study power, which could not be obtained in more searchable data but in a limited number of patients from a single center. Moreover, NIS does not contain clinical and physiological data on ICH volume, location, intraventricular extension or level of consciousness. It is also worth noting that the NIS sample only accounts for events occurring during admission.

\section{Conclusions}

Both IS and ICH are strong predictors of mortality in LVAD patients. The CCI is a strong predictor of both early IS and ICH in patients who undergo LVAD placement. Younger patients with LVAD are at greater risk for ICH than older patients, although the reason remains unclear. Renal disease, liver disease and coagulation disorder are independent predictors of mortality in LVAD patients with IS. In summary, comorbidity burden is a strong predictor of both IS and ICH in patients who undergo LVAD placement and can be used as a predictor and screening tool for LVAD placement.

\section{Acknowledgements}

The authors are grateful to Jennifer Pfaff and Susan Nord of Aurora Cardiovascular Services for editorial preparation of the manuscript.

\section{Footnote}

Conflicts of Interest: The authors have no conflicts of interest to declare.

Ethical Statement: Since the database is publically available and has de-identified data, this study was found to be IRB exempt and does not require informed consent.

\section{References}

1. Benjamin EJ, Blaha MJ, Chiuve SE, et al. Heart Disease and Stroke Statistics-2017 Update: A Report From the American Heart Association. Circulation 2017;135:e146-e603.

2. Fang JC. Rise of the machines--left ventricular assist devices as permanent therapy for advanced heart failure. $\mathrm{N}$ Engl J Med 2009;361:2282-5.

3. Miller LW, Guglin M. Patient selection for ventricular assist devices: a moving target. J Am Coll Cardiol 2013;61:1209-21.

4. Kirklin JK, Naftel DC, Pagani FD, et al. Seventh INTERMACS annual report: 15,000 patients and counting. J Heart Lung Transplant 2015;34:1495-504.

5. Kirklin JK, Naftel DC, Pagani FD, et al. Sixth INTERMACS annual report: a 10,000-patient database. J Heart Lung Transplant 2014;33:555-64.

6. Kirklin JK, Naftel DC, Kormos RL, et al. Fifth INTERMACS annual report: risk factor analysis from more than 6,000 mechanical circulatory support patients. J Heart Lung Transplant 2013;32:141-56.

7. Tsukui H, Abla A, Teuteberg JJ, et al. Cerebrovascular accidents in patients with a ventricular assist device. J Thorac Cardiovasc Surg 2007;134:114-23.

8. John R, Panch S, Hrabe J, et al. Activation of endothelial and coagulation systems in left ventricular assist device recipients. Ann Thorac Surg 2009;88:1171-9.

9. Eckman PM, John R. Bleeding and thrombosis in patients with continuous-flow ventricular assist devices. Circulation 2012;125:3038-47.

10. Kurien S, Hughes KA. Anticoagulation and bleeding in patients with ventricular assist devices: walking the tightrope. AACN Adv Crit Care 2012;23:91-8.

11. Boyle AJ, Jorde UP, Sun B, et al. Pre-Operative Risk Factors of Bleeding and Stroke During Left Ventricular 
Assist Device Support. J Am Coll Cardiol 2014;63:880-8.

12. Whitson BA, Eckman P, Kamdar F, et al. Hemolysis, Pump Thrombus, and Neurologic Events in Continuous-Flow Left Ventricular Assist Device Recipients. Ann Thorac Surg 2014;97:2097-103.

13. Morgan JA, Brewer RJ, Nemeh HW, et al. Stroke while on long-term left ventricular assist device support: incidence, outcome, and predictors. ASAIO J 2014;60:284-9.

14. Willey JZ, Gavalas MV, Trinh PN, et al. Outcomes after stroke complicating left ventricular assist device. J Heart Lung Transplant 2016;35:1003-9.

15. Teuteberg JJ, Slaughter MS, Rogers JG, et al. The HVAD Left Ventricular Assist Device: Risk Factors for Neurological Events and Risk Mitigation Strategies. JACC Heart failure 2015;3:818-28.

16. Charlson ME, Pompei P, Ales KL, et al. A new method of classifying prognostic comorbidity in longitudinal studies: development and validation. J Chronic Dis 1987;40:373-83.

17. de Groot V, Beckerman H, Lankhorst GJ, et al. How to measure comorbidity. a critical review of available methods. J Clin Epidemiol 2003;56:221-9.

18. Sarfati D, Tan L, Blakely T, et al. Comorbidity among patients with colon cancer in New Zealand. N Z Med J 2011;124:76-88.

19. Mnatzaganian G, Ryan P, Norman PE, et al. Accuracy of hospital morbidity data and the performance of comorbidity scores as predictors of mortality. J Clin Epidemiol 2012;65:107-15.

20. Frontera JA, Starling R, Cho SM, et al. Risk factors, mortality, and timing of ischemic and hemorrhagic stroke with left ventricular assist devices. J Heart Lung Transplant 2017;36:673-83.

21. Meyer AL, Malehsa D, Bara C, et al. Acquired von

Cite this article as: Shahreyar M, Bob-Manuel T, Khouzam RN, Bashir MW, Sulaiman S, Akinseye O, Sharma A, Carter A, Latham S, Bhandari S, Jahangir A. Trends, predictors and outcomes of ischemic stroke and intracranial hemorrhage in patients with a left ventricular assist device. Ann Transl Med 2018;6(1):5. doi: 10.21037/atm.2017.12.23
Willebrand syndrome in patients with an axial flow left ventricular assist device. Circ Heart Fail 2010;3:675-81.

22. Wever-Pinzon O, Naka Y, Garan AR, et al. National trends and outcomes in device-related thromboembolic complications and malfunction among heart transplant candidates supported with continuous-flow left ventricular assist devices in the United States. J Heart Lung Transplant 2016;35:884-92.

23. Sacco RL, Boden-Albala B, Gan R, et al. Stroke incidence among white, black, and Hispanic residents of an urban community: the Northern Manhattan Stroke Study. Am J Epidemiol 1998;147:259-68.

24. Morgenstern LB, Smith MA, Lisabeth LD, et al. Excess stroke in Mexican Americans compared with non-Hispanic Whites: the Brain Attack Surveillance in Corpus Christi Project. Am J Epidemiol 2004;160:376-83.

25. Harvey L, Holley C, Roy SS, et al. Stroke After Left Ventricular Assist Device Implantation: Outcomes in the Continuous-Flow Era. Ann Thorac Surg 2015;100:535-41.

26. van den Bergh WM, Lansink-Hartgring AO, van Duijn AL, et al. Thromboembolic stroke in patients with a HeartMate-II left ventricular assist device - the role of anticoagulation. J Cardiothorac Surg 2015;10:128.

27. Englert JA 3rd, Davis JA, Krim SR. Mechanical Circulatory Support for the Failing Heart: ContinuousFlow Left Ventricular Assist Devices. Ochsner J 2016;16:263-9.

28. Slaughter MS, Rogers JG, Milano CA, et al. Advanced heart failure treated with continuous-flow left ventricular assist device. N Engl J Med 2009;361:2241-51.

29. Yoshihara H, Yoneoka D. Understanding the statistics and limitations of large database analyses. Spine 2014;39:1311-2. 
Supplementary

ICD-9 CM codes

37.60 Implantation or insertion of biventricular external heart assist system

37.62 Insertion of temporary non-implantable extracorporeal circulatory assist device

37.64 Removal of external heart assist system(s) or device(s)

37.65 Implant of single ventricular (extracorporeal) external heart assist system

37.66 Insertion of implantable heart assist system

37.68 Insertion of percutaneous external heart assist device

ICD-9 CM codes intracranial hemorrhage

430, 431, 432.1, 432, 432.9

ICD-9 CM codes of stroke

$433,434,434.1,434.9,435,436$ 